GARCÍA CARMONA, D. “eSports: origen, presente y futuro”, REDUR 19, diciembre 2021, págs. 109-117. ISSN 1695078X. https://doi.org/10.18172/redur.5127

\title{
ESPORTS: ORIGEN, PRESENTE Y FUTURO
}

\author{
DAVID GARCÍA CARMONA \\ TUtOR DE LA FACUlTAD DE DERECHO \\ UNIVERSIDAD INTERNACIONAL DE LA RIOJA (UNIR) \\ dgc_skater@hotmail.com
}

SUMARIO: I. INTRODUCCIÓN: CONCEPTO DE ESPORT. II. ORIGEN, EVOLUCIÓN Y LLEGADA A ESPAÑA DE LOS DEPORTES ELECTRÓNiCOS. III. ACTUALIDAd DE LOS ESPORTS. IV. PRESENTE DE los ESPORTS EN EsPaña. V. CONTROVERSia Y REGULACión DE los ESPORTS EN ESPAÑA. VI. DERECHO COMPARADO. VII. CONCLUSIONES. VIII. BIBLIOGRAFÍA

RESUMEN: Los eSports forman parte de una industria que está en pleno auge y crecimiento, siendo uno de los grandes nichos de mercado en nuestra actualidad, a pesar de la pandemia mundial ocasionada por la Covid19. Prueba de ello, es que jugadores profesionales tanto de Fútbol como de Baloncesto están invirtiendo en equipos profesionales de eSports, formando los suyos propios e incluso creando competiciones de deportes electrónicos.

PALABRAS CLAVE: eSports, Derecho Deportivo, legislación, videojuegos, actualidad.

\section{ESPORTS: ORIGIN, PRESENT AND FUTURE}

ABSTRACT: ESports are part of an industry that is booming and growing, being one of the great market niches today despite the global pandemic caused by Covid-19. Proof of this is that professional soccer and basketball players are investing in professional eSports teams, forming their own and even creating electronic sports competitions.

KEYWORDS: E-Sports, Sports Law, legislation, videogames, news. 


\section{Introducción: concepto de eSport}

Los eSports o deportes electrónicos son competiciones profesionales que se ubican en el sector del entretenimiento y, en concreto, dentro de la industria de los videojuegos. Estas competiciones suelen desarrollarse a partir de videojuegos de estrategia en tiempo real, disparos en primera persona y arenas de batalla multijugador en línea.

Según el diccionario informático Techlib: “eSports (pronunciado 'e-sports') es un término general utilizado para describir las competiciones de videojuegos. Al igual que los eventos deportivos, los juegos de eSports a menudo se juegan ante audiencias en vivo y pueden transmitirse por Internet también."1

A su vez, en España, la Asociación Española de Videojuegos (AEVI) determina que eSport "es el nombre con el que popularmente se conocen a las competiciones de videojuegos estructuradas a través de jugadores, equipos, ligas, publishers, organizadores, broadcasters, patrocinadores y espectadores. Se puede jugar de forma amateur o profesionalizada y de forma presencial u online.”2

\section{Origen, evolución y llegada a España de los deportes electrónicos}

Los eSports, como competiciones de videojuegos a gran escala, tuvieron su primer gran torneo en 1980 y contaron con más de 10.000 participantes, de forma presencial, de todo Estados Unidos. El juego protagonista de tal competición era el Space Invaders, más conocido como "mata marcianos".

Desde esta primera gran aparición de los eSports en un evento multitudinario hasta la actualidad, se han celebrado otros eventos en diversas décadas, como en la de los noventa, destacando en los noventa, el Nintendo World Championship, cuyos protagonistas principales fueron el archiconocido Super Mario y The Legend of Zelda. Posteriormente, desde 1997 se comenzaron a celebrar los torneos de Red Annibilation y Starcraft, ambos considerados como eSports modernos. Los dos pasaron a la historia de esta modalidad de entretenimiento por motivos muy diferentes, en concreto, el primero lo hizo por tener como premio para el ganador o ganadora un Ferrari, y el segundo por considerar a los jugadores como atletas, es decir, jugadores profesionales.

En Europa, se fundó la Electronic Sports League (ESL) en el año 2000 y mediante ella llegaron las competiciones de eSports a España. En el año 2006 se celebró, en un cibercafé cercano a Plaza de España en Madrid, la quinta edición de la e-Sensation, que serviría para elegir a la selección española que participaría en la European Nations Championship de ESL. Tras ello, la e-Sensation fue comprada por ESL y pasó a ser ESL España.

El punto álgido mundial de este fenómeno llegó de la mano de Bližard con el Warcraft hasta llegar al League Of Legends, considerado a día de hoy el juego más popular de eSports. ${ }^{3}$

\section{Actualidad de los eSports}

La evolución de los videojuegos ha pasado de un uso principalmente individual a un uso colectivo y ello se debe al desarrollo de las nuevas tecnologías, lo que, a su vez ha propiciado que

\footnotetext{
${ }^{1}$ Información obtenida de la página de Techlib, https://techlib.net/definition/esports.html

2 http://www.aevi.org.es/e-sports/

${ }^{3}$ Infinity Talent. (2021). Olimpiadas Intergalácticas. El origen de los esports. 2021, de Infinity Talent. Sitio web: https://infinitytalent.es/la-historia-de-los-esports/
} 
muchos usuarios tengan acceso a ellos. Asimismo, dicho desarrollo tecnológico ha favorecido la creación de competiciones, las cuales son vistas y disfrutadas por millones de personas, y más aún en un contexto de pandemia mundial, generando, por ende, unas importantes cantidades de beneficios y produciendo finalmente una profesionalización de los competidores en estos eventos.

Es un sector en auge y en constante y sostenible crecimiento tal y como indican consultoras especializadas en el mismo, entre las que cabe destacar $N e w 200^{4}$, que demuestra un crecimiento progresivo desde 2018 y prevé alcanzar un margen de beneficio de más de 1500 millones de euros en 2025.

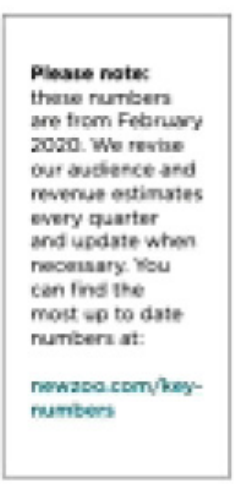

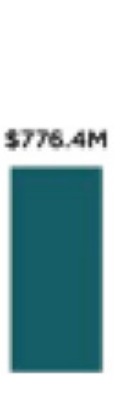

2008

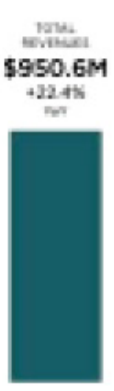

2009

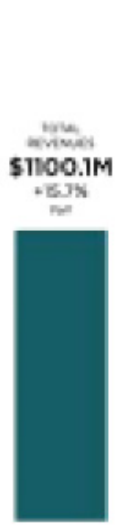

2000

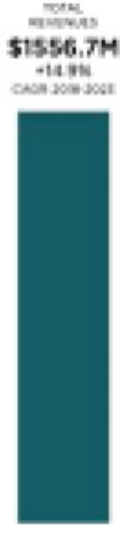

20025

Las fuentes para la obtención de estos beneficios no proceden exclusivamente del número de espectadores. De hecho, la principal fuente de ingresos de este sector proviene de los contratos de patrocinio de diversas marcas a los equipos profesionales de jugadores, así como a los propios eventos de eSports. Otras fuentes de ingresos del sector son el "merchandising", la adquisición de productos por parte de los consumidores tales como sudaderas, camisetas, tazas...; [lo que obtienen los "publishers", es decir, el beneficio por la explotación de los derechos de autor sobre los videojuegos]; los derechos de retransmisión de estos eventos, etc.

Varias figuras relevantes del mundo del deporte a nivel internacional (además de las reflejadas en el apartado IV del presente artículo), como el jugador argentino del Fútbol Club Barcelona, Sergio Agüero, apuestan por este sector. De hecho, dicho futbolista ha creado recientemente su propio equipo profesional de eSports, llamado KRÜsports con el que pretende colaborar con la plataforma Twitch $^{5}$ para hacer torneos y sorteos de sus principales patrocinadores ${ }^{6}$.

El último gran hito de este sector han sido las Olimpiadas de eSports. El Comité Olímpico Internacional (COI, en adelante) ha anunciado la creación de su primera competición de deportes

\footnotetext{
${ }^{4}$ Es necesario indicar que esta empresa, además de publicar estudios periódicos sobre el nivel de ingresos de la industria, también ofrece, como se aprecia en la tabla, proyecciones del negocio en los años venideros.

5 Plataforma que permite realizar trasmisiones en vivo, propiedad de la empresa Amazon Inc. www.twitch.tv

${ }^{6}$ Redacción Marca. (2020). KRÜ el equipo de eSports del Kun Agüero ya es una realidad. MARCA. (Recuperado de: https://www.marca.com/videojuegos/lo-mas-gaming/2020/10/15/5f88a43722601d04138b45d0.html)
} 
electrónicos, las Series Olímpicas Virtuales (OVS), con la colaboración de cinco federaciones internacionales: béisbol, ciclismo, remo, vela y automovilismo.

Estos juegos virtuales se desarrollaron del 13 de mayo al 23 de junio de 2021 y, según anunció dicho organismo "movilizarán a los entusiastas de los deportes virtuales, los deportes electrónicos y los juegos en todo el mundo, para llegar a nuevas audiencias olímpicas, a la vez que fomentarán el desarrollo de formas físicas y no físicas de deporte en línea con las recomendaciones de la Agenda Olímpica 2020+5 del COI."

\section{Presente de los eSports en España}

Según Newroo, España es el décimo país del mundo por ingresos de la industria del videojuego, contando con 2,656.000 dólares de ingresos ${ }^{7}$ en el año 2020. Además de ello, cuenta con su propia competición nacional, organizada por la Liga de Videojuegos Profesional (LVP). Grandes marcas internacionales como Vodafone o Nike empiezan a patrocinar a los equipos profesionales de este sector del entretenimiento.

Asimismo, Broadband Savvy, empresa de Reino Unido experta en banda ancha, en su último estudio sobre las mejores y peores ciudades a nivel mundial para desarrollar actividades competitivas en gaming y en eSports sitúa a Barcelona en el puesto 8 del top 10 del ranking.

Haciendo un inciso en el nicho de mercado y en la oportunidad de negocio que suponen los eSports en nuestro país, jugadores profesionales de fútbol, tales como Dani Parejo (jugador del Villarreal), Álvaro Morata (jugador de la Juventus) y jugadores profesionales de baloncesto como Álex Abrines (jugador del F.C. Barcelona de baloncesto) invierten dinero apoyando a determinados equipos de este sector, en concreto, estos tres jugadores invierten en el equipo profesional de eSports valenciano Ramboot. ${ }^{9}$

Sin embargo, ellos no son los primeros en invertir en esta industria en auge. Uno de los pioneros en nuestro país es el famoso futbolista Gerard Piqué, quien fundó en el año 2016 una empresa de eSports denominada eFootball con una voluntad de negocio clara: promover y crear contenidos de juegos. Además de la iniciativa de la creación de empresa, el futbolista junto con la compañía de videojuegos japonesa Konami anunciaron hace unos años un acuerdo para crear una competición profesional de eSports poniendo el foco en el videojuego PES2018 (Pro Evolution Soccer). Esta liga es de carácter internacional y ambas empresas colaborarán a su vez con una tercera, DeSports, que se encargará de expandir la competición por el continente asiático. ${ }^{10}$

\section{Controversia y regulación de los eSports en España}

Con estos datos, en un sector en auge con una ingente cantidad de beneficios, se está ante un paradigma y un constante debate: ¿es necesario una regulación del sector o no?

\footnotetext{
7 Newzoo. (2020). Top 10 Countries/Markets by Game Revenues. 2020, de Newzoo. Sitio web: https://newzoo.com/insights/rankings/top-10-countries-by-game-revenues/

8 https://broadbandsavvy.com/best-cities-for-gamers/

${ }^{9}$ Merino, A. (2019). "Morata, Parejo y Abrines se meten en los esports con su propio equipo". AS. (Recuperado de: https://esports.as.com/industria/Alvaro-Morata-esports-propio-equipo_0_1301869810.html)

10 Redacción Crónica Global. (2017). Gerard Piqué crea una liga de 'e-sports' con Konami. Crónica Global. (Recuperado de: https://cronicaglobal.elespanol.com/deportes/gerard-pique-e-sports-konami_99614_102.html)
} 
a) Por un lado, existe un sector que aprecia motivos evidentes para someter a esta industria a una regulación tales como verificar la solvencia de las empresas que intervienen en dicho ámbito, velar por la seguridad de los menores y otros motivos que se exponen más adelante.

b) Por otro lado, existe un sector cuyo gran defensor en España es la AEVI (Asociación Española de Videojuegos) ${ }^{11}$, que apoya la no regulación pues determinan que de hacerla se pondrían trabas y limitaciones al crecimiento de un sector próspero, que tiene posibilidades de ofrecer puestos de trabajo de calidad, entre otros muchos beneficios.

Dentro de la primera postura, es decir, de cara a poder establecer una regulación de los eSports es necesario analizar un aspecto o consideración fundamental de los mismos y es si son o no deportes y si los competidores profesionales desempeñan una actividad deportiva.

Un paso muy importante en este sentido lo ha dado el COI, pues se ha planteado incluir esta modalidad dentro de su carta olímpica atendiendo a dos factores fundamentales:

- "Los eSports están mostrando un fuerte crecimiento, especialmente entre los jóvenes de diferentes países, y pueden proporcionar una plataforma compatible con el Movimiento Olímpico.”

- ' 'La competición dentro de los eSports puede ser considerada una 'actividad deportiva', los jugadores se preparan y entrenan con una intensidad que puede compararse a la de los atletas de los deportes tradicionales." 12

No obstante, este organismo indica que para incluirlos dentro de su carta olímpica sería necesaria la adopción de ciertas medidas, como no infringir los valores olímpicos, la existencia de una organización que garantice y vele por el cumplimiento de las reglas del Movimiento Olímpico (como antidoping, apuestas), etc.

Otro punto de inflexión es que si bien hay videojuegos que cumplen con los valores olímpicos (Tennis World Tour, NBA 2k, FIFA...), existen otros que no los cumplen por contener violencia explícita en algunos casos o por otros motivos y, por ello, tendrían que ser suprimidos, como el LOL (League of Legends) o el CoD (Call of Duty).

Otros aspectos de especial importancia a tener en cuenta para una posible regulación de esta modalidad en nuestro país son:

- La fiscalidad.

- La protección de los menores.

- La consideración de los jugadores profesionales como trabajadores.

- La protección de los consumidores y usuarios.

- La protección de los derechos de imagen y derechos de propiedad intelectual.

\footnotetext{
${ }^{11}$ Información extraída de la página web oficial de AEVI, www.aevi.org.es

12 Ayora, V. (2019). "El COI acepta a los eSports como 'actividad deportiva". MARCA. (Recuperado de: https://www.marca.com/esports/2019/04/06/5ca8f65c22601dfd0a8b463a.html)
} 
Al margen de esta consideración de si son deporte o no, hay que analizar otras grandes diferencias con los deportes históricos:

1. Los derechos sobre la propiedad intelectual: para practicar la mayoría de los deportes, como el baloncesto, el tenis, el fútbol, etc., no hay que pagar al inventor de dichos deportes; sin embargo, en esta modalidad sí, pues como he señalado previamente existe un creador de estos contenidos al que hay que pagar para poder desarrollar esta actividad. ${ }^{13}$

2. No existe una actividad física: esto para muchos Estados es un hándicap, pues lo consideran como el principal óbice para determinar los esports como deporte y poder elaborar una regulación adecuada.

Pese a ello, este hecho no debiera ser un óbice, pues sin ir más lejos el ajedrez es un deporte, así lo considera el COI y posee su propia Federación, la FIDE (acrónimo francés Fédération Internationale des Échecs).

Actualmente en España no existe legislación específica que dé cabida a este sector sino que se tratan en diversos textos legislativos de aspectos relacionados con los eSports, como en la Ley 7/2010, de 31 de marzo, General de la Comunicación Audiovisual, en relación con los derechos de retransmisión, en la Ley 34/1988, de 11 de noviembre, General de Publicidad, en relación con el patrocinio, o en el Real Decreto Legislativo 1/1996, de 12 de abril, por el que se aprueba el texto refundido de la Ley de Propiedad Intelectual, entre otros.

No obstante, ha habido en nuestro país un intento para que los deportes electrónicos tuvieran cabida legal. En concreto, se propuso en las Islas Canarias y se pretendía incluir a los deportes electrónicos en la nueva Ley Canaria del Deporte. Con posterioridad, dicho proyecto no prosperó en el Parlamento Canario.

A pesar de esta primera experiencia autonómica, en la que los deportes electrónicos no vieron la luz, el pasado 12 de mayo se publicó en el Boletín Oficial de las Cortes Generales, la Proposición no de Ley para incentivar la industria del videojuego en España ${ }^{14}$.

En dicha propuesta se recogen una serie de medidas con la finalidad de atraer tanto capital como proyectos extranjeros, mantener la industria en España creando puestos de trabajo, así como incentivar la creación de startups para el desarrollo y comercialización de nuevos proyectos. Algunas de estas medidas son:

- Reconocer el sector del videojuego como una industria emergente.

- Modificar el artículo 36 de la Ley del Impuesto sobre Sociedades para incluir el videojuego en la deducción por inversiones en producciones cinematográficas, series audiovisuales y espectáculos.

- Crear un órgano consultivo de análisis, asesoramiento y apoyo: Grupo Asesor del videojuego.

\footnotetext{
${ }^{13}$ Los programas de ordenador son propiedad intelectual y están protegidos por derechos de autor. Art. 10.1.i) del Real Decreto Legislativo 1/1996, de 12 de abril, por el que se aprueba el texto refundido de la Ley de Propiedad Intelectual, regularizando, aclarando y armonizando las disposiciones legales vigentes sobre la materia.

${ }^{14}$ Boletin Oficial de las Cortes Generales. Congreso de los Diputados XIV Legislatura. Serie D General, núm. 270 de 12 de mayo de 2021, págs. 85 y ss. https://www.congreso.es/public_oficiales/L14/CONG/BOCG/D/BOCG-14D-270.PDF
} 
- Desarrollar un programa específico de apoyo a la industria de los videojuegos.

- Diseñar un plan de comercialización, captación de inversiones y promoción internacional de la industria española del videojuego como Marca España a través del ICEX (Instituto del Comercio Exterior).

\section{Derecho Comparado}

Como breve inciso de Derecho comparado, hay que referirse a nuestros países vecinos:

En primer lugar, Francia pues ha sido el primer país en Europa en regular los eSports. Lo ha hecho en los artículos 101 y 102 de su Loi no 2016-1321 du 7 octobre 2016 pour una République numérique ${ }^{15}$. El primero de estos artículos establece una definición de qué es un videojuego y qué es una competición de videojuegos, mientras que el segundo ofrece un concepto de jugador profesional de deportes electrónicos: "El jugador profesional asalariado de videojuegos competitivo se define como toda persona cuya actividad remunerada sea la participación en concursos de videojuegos en una relación de subordinación legal con una asociación o empresa que cuente con la aprobación del ministro encargado de lo digital, especificado por vía reglamentaria.”.

Además de estos aspectos, dichos artículos regulan otros, como la duración máxima de la relación contractual, contenidos mínimos para la validez del contrato, expresa prohibición de las cláusulas de rescisión unilaterales, etc.

En segundo lugar, es necesario destacar el Principado de Andorra pues ya tiene publicado un proyecto de ley de deportes electrónicos ${ }^{16}$, es decir, a diferencia de Francia no es una regulación.

En la exposición de motivos de dicho proyecto de Ley se determina un marco jurídico vinculado a varios objetivos, como dotar de seguridad jurídica la actividad que se genera en el espacio digital, asegurar la protección de los consumidores, atraer inversión y empresas extranjeras, favorecer el tejido empresarial en el país y fomentar la innovación y la creatividad. Todo esto requiere un marco jurídico sólido, previsible y, sobre todo, capaz de responder al dinamismo y al cambio permanente del sector, de la tecnología, de las prácticas empresariales y de las costumbres sociales.

También se contemplan en dicho texto, en concreto en el artículo 2, diversas definiciones, como la de deportes electrónicos: "Toda competición o acontecimiento abierto al público y que se basa en videojuegos, en el que participan de forma presencial o remota dos o más jugadores y en el que se ofrecen premios a los jugadores atendiendo al resultado de los juegos correspondientes y a las normas preestablecidas para los organizadores para regular las competiciones o acontecimiento mencionado." 17

Además, dicho precepto establece con detalle diferencias entre clubs profesionales y clubs no profesionales de deportes electrónicos, entre jugadores profesionales y jugadores no profesionales, entre otros.

\footnotetext{
15 www.legifrance.gouv.fr

16 Butlletí del Consell General - núm. 45/2020 - Casa de la Vall, 17 de juny del 2020- (Recuperado de: http://www.consellgeneral.ad/ca/activitat-parlamentaria/iniciatives-legislatives/projectes-de-llei/projecte-dellei-d2019esports-electronics)

${ }_{17}$ Barbarà, A. (2021). El proyecto de ley para regular los esports en Andorra. 2021, de www.alexbarbarbara.es Sitio web: https://www.alexbarbara.es/ley_esports_andorra/
} 
De la lectura de dicho precepto se determina que, para que un jugador sea profesional, debe estar contratado por un club profesional eSports, pues se define al jugador profesional como a todo aquel residente legal en el territorio andorrano, mayor de 15 años, que esté habilitado para participar en competiciones de eSports y reciba una remuneración de un club para hacerlo.

Dicho proyecto, si bien no es muy extenso (pues contiene solo cincuenta artículos, dos disposiciones transitorias y dos disposiciones finales), recoge aspectos esenciales además de los señalados anteriormente, como las obligaciones tanto de los clubs profesionales de deportes electrónicos como las de los no profesionales, la forma jurídica de ambos tipos de clubs y los requisitos para organizar competición de deportes electrónicos.

En tercer lugar, es preciso destacar a Alemania como uno de los pioneros de Europa en este sector. Si bien no tiene una legislación específica que lo aborde y lo regule, alberga alguna de las empresas más grandes a nivel europeo de esta industria como la muy conocida eSports-Bund Deutschland (ESBD) que aúna bajo su tutela a más de 20 empresas dedicadas exclusivamente a los deportes electrónicos. Otro aspecto a resaltar del país germano es que los eSports se incluyeron en el pacto de gobierno a raíz de las últimas elecciones celebradas donde se expone que: "en el futuro reconoceremos a los deportes electrónicos como un deporte propio con derechos de asociación específicos y los ayudaremos a crecer en una perspectiva olímpica.".

A modo de suma de este apartado, estos tres países son pioneros en nuestro continente en la contemplación y aprovechamiento de los deportes electrónicos. Francia al incluirlos directamente en su ordenamiento jurídico, Andorra al establecer un proyecto para regular los deportes electrónicos, dinamizado por la llegada de "youtubers" y creadores de contenido a su país, y Alemania al incluirlo en pactos políticos de Gobierno.

\section{Conclusión}

A modo de conclusión, cabe destacar que la industria de los videojuegos como mercado emergente plantea numerosas cuestiones controvertidas sobre su, funcionamiento. Es por ello que considero necesaria una regulación de los eSports en España. Hay aspectos destacados con anterioridad, como la protección de los menores, la fiscalidad, la protección de los consumidores, entre otros, que deben abordarse para dar seguridad a todos los componentes de la industria.

Siendo España uno de los países más consumidores de esta industria a nivel mundial y también uno de los países con mayor previsión de desarrollo del sector tal como indican organismos y consultoras especializadas, estimo necesaria una regulación del mismo que bien puede llegar a través de iniciativas como la ya mencionada Proposición no de Ley.

El Derecho ha de ajustarse a los cambios que se producen en la sociedad. Debido a esta máxima, aprovechando el auge y la gran evolución experimentada, y las previsiones de desarrollo de esta industria, hay que regular este sector.

Sin embargo, no considero necesario un intervencionismo exacerbado, pues sería un lastre para una industria que puede ser muy beneficiosa para nuestra sociedad ya que crea puestos de trabajo, atrae capital y proyectos internacionales, etc. Esta creación de empleo a su vez dotaría de dinamismo a la economía, pues muchos jóvenes podrían encontrar su futuro profesional en este sector, bien como profesionales bien en los puestos derivados del mismo, obteniendo así independencia económica y contribuyendo a la economía estatal con consumo y pago de impuestos. 


\section{Bibliografía}

AYORA, V., "El COI acepta a los eSports como 'actividad deportiva", Marca, 6 de abril de 2019. (https://www.marca.com/esports/2019/04/06/5ca8f65c22601dfd0a8b463a.html)

BARBARÀ, A., "El proyecto de ley para regular a los deportes electrónicos en Andorra”, Alex Barbará [blog], 12 de febrero de. 2021, (https://www.alexbarbara.es/ley_esports_andorra/)

INFINITY TALENT, "Esports", Infinity Talent [página web], 26 de marzo de 2021 (https://infinitytalent.es/la-historia-de-los-esports/\#: :text=Tenemos $\% 20$ que $\% 20$ remontarnos $\% 20 \mathrm{a} \% 201$ a,competici $\% \mathrm{C} 3 \% \mathrm{~B} 3 \mathrm{n} \% 20 \mathrm{de} \% 20$ videojuegos $\% 20 \mathrm{o} \% 20$ eSport s.\&text $=\mathrm{En} \% 201972 \% 20 \mathrm{en} \% 20 \mathrm{la} \% 20$ Universidad, $\% \mathrm{E} 2 \% 80 \%$ Colimpiadas $\% 20$ Inter gal $\% \mathrm{C} 3 \%$ A1cticas $\%$ E2\%80\%9D $\% 20 \% \mathrm{C} 2 \%$ BFPremio $\% 3 \mathrm{~F}$ )

MERINO, A. "Morata, Parejo y Abrines se meten en los esports con su propio equipo", $A s, 19$ de noviembre de 2019. (https://esports.as.com/industria/Alvaro-Morata-esports-propioequipo_0_1301869810.html)

REDACCIÓN CRÓNICA GLOBAL, "Gerard Piqué crea una liga de 'e-sports' con Konami”, Crónica Global, 13 de noviembre de 2017 (https://cronicaglobal.elespanol.com/deportes/gerard-piquee-sports-konami_99614_102.html)

REDACCIÓN MARCA, "KRÜ el equipo de eSports del Kun Agüero ya es una realidad”, Marca, 16 de octubre de 2020 (https://www.marca.com/videojuegos/lo-mas-gaming/2020/10/15/ 5f88a43722601d04138b45d0.html) 